\title{
Local and global mechanisms of one- and two-dimensional orientation illusions
}

\author{
PETER WENDEROTH and RICK VAN DER ZWAN \\ University of Sydney, Sydney, New South Wales, Australia
}

\begin{abstract}
One-dimensional (1-D) orientation illusions induced on a test grating by a tilted and surrounding 1-D inducing grating have a well-known angular function that exhibits both repulsion and attraction effects. Two-dimensional (2-D) orientation illusions are those induced on a test grating by 2-D image modulation, such as a pair of superimposed inducing gratings at different orientations, usually orthogonal (a plaid). Given the known angular functions induced by the plaid component gratings, two hypotheses were developed that predicted different plaid-induced illusion functions. Hypothesis 1 states that the 1-D component-induced effects simply add linearly; Hypothesis 2 states that there is an additional mechanism that responds to the virtual axes of mirror symmetry of the plaid and adds to the effect. The data of two experiments were consistent with the predictions from the second hypothesis but not the first. Possible neural substrates of mechanisms that extract axes of symmetry are discussed; it is suggested that such global symmetry axes may underlie the perceived orientation of complex shapes.
\end{abstract}

The perceived orientation of a test grating is altered when it is surrounded by a single or one-dimensional (1-D) inducing grating at a different orientation. The direction of this orientation illusion varies with inducing tilt, and it has been suggested that the directionally different segments of the angular function reflect the operation of different mechanisms. Specifically, we showed that spatial differences between test and inducing stimuli reduced one component effect but not the other and that an upright square surrounding the whole display eradicated the latter but had no effect on the former (Wenderoth \& Johnstone, 1988a). This study is designed to examine the effects of similar manipulations on orientation illusions in which a test grating is surrounded by $2-\mathrm{D}$ modulation consisting of a pair of orthogonally oriented gratings (a plaid). Two sets of predictions were developed and tested: one in which the predicted plaid effect is the linear sum of the 1-D component processes and another in which it is the linear sum of the component processes plus an additional process. The strategy was to develop hypothetical

\footnotetext{
This research was supported in part by Australian Research Council Grant 78831871 to the first author, and it provided for the research position of the second author. Thanks are due Senior Systems Analyst John Holden for all aspects of assistance with computing facilities. A draft of this paper was prepared while P.W. was on study leave at the SmithKettlewell Eye Research Institute, San Francisco, CA, September 7December 6, 1990, and a version of it was presented at the 31 st Annual Meeting of the Psychonomic Society, New Orleans, November, 1990. Thanks are due the University of Sydney for financial support, the SmithKettlewell Institute for accommodation, and Christopher Tyler for the use of facilities and for his invaluable suggestions regarding the manner in which to present the arguments in quantitative fashion. Correspondence should be addressed to Peter Wenderoth, Department of Psychology, University of Sydney, Sydney, NSW 2006, Australia.
}

functions, modeled on real data and then to select inducing orientations most likely to discriminate between the alternate hypotheses.

Gibson and Radner (1937) showed that a subjectively vertical line no longer appeared vertical when it was presented after adaptation to a tilted line. For similar line and grating orientations, this aftereffect was one of repulsion, such that the test line's orientation appeared pushed away from the inducing orientation. However, when test and inducing stimuli differed in orientation by almost $90^{\circ}$, the effect reversed direction, such that the test line's orientation appeared pulled toward the inducing grating. Gibson referred to the repulsion effect as the direct effect, because it represented the interaction of two stimuli, both of which were at or near vertical. He termed the smaller attraction effect the indirect effect, because it represented the interaction of near horizontal on vertical contours. That is, direct effects were those that occurred with test and inducing stimuli at similar orientations; indirect effects were those that occurred between stimuli at close to orthogonal orientations. Thus, a subjectively horizontal stimulus exhibits direct effects when presented subsequently to a near-horizontal inducing stimulus and a smaller, orientationally opposite indirect effect when the inducing stimulus is near vertical (Morant \& Harris, 1965). Gibson (1937) also showed that a simultaneous tilt illusion occurs when a test line is superimposed on a tilted inducing grating. It has since been demonstrated that direct and indirect tilt illusions and aftereffects occur when both test and inducing stimuli are gratings (e.g., Campbell \& Maffei, 1971; Mitchell \& Muir, 1976), that both the aftereffect and the illusion occur with the test stimulus at the oblique meridian (Mitchell \& Muir, 1976 ; O'Toole \& Wenderoth, 1977), and that the tilt illusion has an angular function similar to that of the tilt aftereffect (O'Toole 


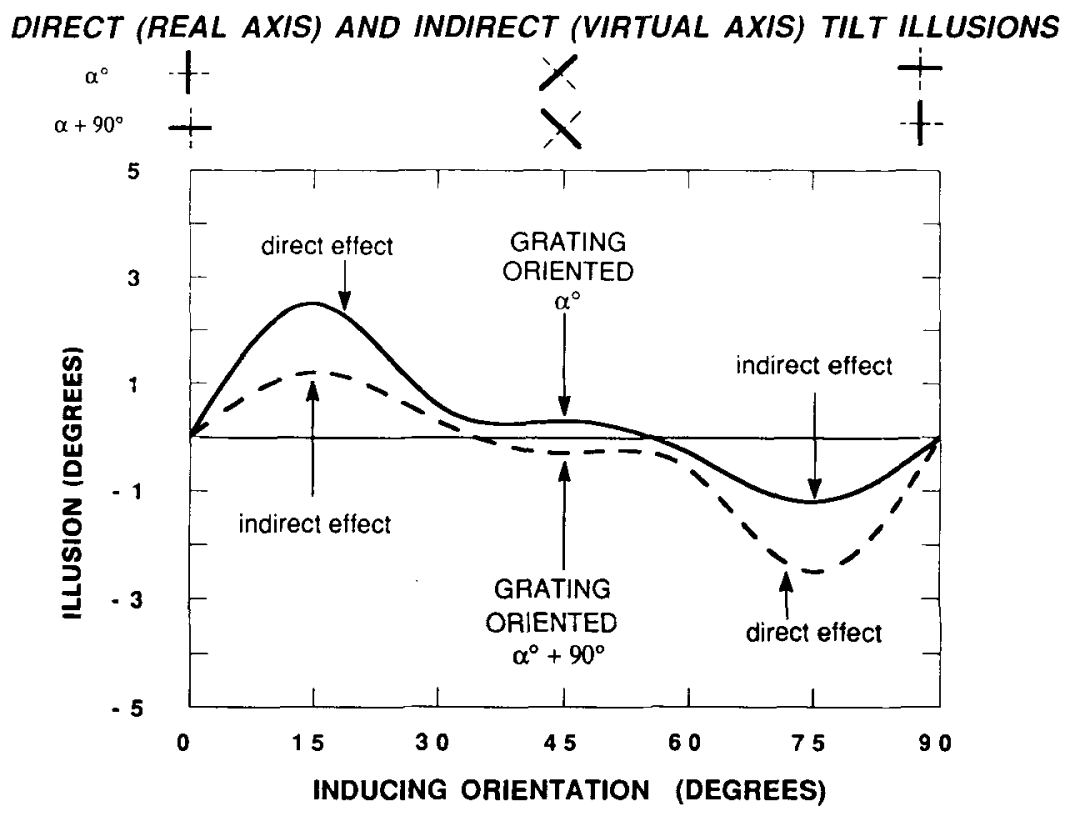

Figure 1. Hypothetical 1-D orientation aftereffect/llusion angular functions induced by single lines or gratings that vary clockwise in orientation from $\alpha^{\circ}$ to $\alpha^{\circ}+90^{\circ}$ (e.g., vertical to horizontal, upper inset) or from $\alpha^{\circ}+90^{\circ}$ to $\alpha^{\circ}$ (e.g., horizontal to vertical, lower inset). For definitions of direct and indirect effects, see text.

\& Wenderoth, 1977; Over, Broerse, \& Crassini, 1972; O'Toole \& Wenderoth, 1977).

The form of the angular function of these orientation aftereffects and illusions is shown schematically in Figure 1. Illusion magnitude, plotted on the ordinate, refers to the observer's point of subjective vertical relative to the point of subjective vertical in the absence of the inducing stimulus. With the test stimulus always vertical, the solid function shows the effect of an inducing line or grating as it tilts clockwise between vertical ( $\alpha^{\circ}$ in inset above Figure 1) and horizontal $\left(\alpha^{\circ}+90^{\circ}\right)$. The dashed function shows the effect on the same vertical test stimulus as an initially horizontal inducing stimulus tilts clockwise from horizontal $\left(\alpha^{\circ}+90^{\circ}\right)$ to vertical $\left(\alpha^{\circ}\right)$.

Blakemore, Carpenter, and Georgeson (1970) attributed the tilt illusion and aftereffect to lateral inhibition between orientation selective channels in V1 visual cortex. Since then, consistent with the theory, other experiments have shown that these effects, like many single cells in V1, are tightly tuned for spatial position and spatial frequency (see Howard, 1982, for summary). For example, the direct tilt illusion is attenuated considerably if the test and inducing components are retinally separated by about $1^{\circ}$ or more (Tolhurst \& Thompson, 1975; Virsu \& Taskinen, 1975; Wallace, 1969) or if the stimuli are spatially contiguous but differ in spatial frequency (Georgeson, 1973). The latter result is also true of the aftereffect (Ware \& Mitchell, 1974).

Wenderoth and Johnstone (1988a) replicated these results for the direct tilt illusion but, for the first time, showed that the indirect effect was completely unaffected by the same variables. In addition, they showed that reduc- ing the width of an inducing annulus surrounding a test grating systematically reduced the direct illusion but had no effect on the indirect effect. Conversely, when the entire test grating with surrounding inducing annulus display was enclosed in a vertical or near-vertical square frame, the direct illusion was unaffected but the indirect effect disappeared. Similar results with a frame were obtained with very briefly flashed stimuli (Wenderoth \& Johnstone, 1988b). These findings led to the conclusion that direct and indirect tilt illusions have different determinants.

Howard (1982, p. 522-523) draws a distinction between the axes of mirror-image symmetry of a shape that are parallel to the main lines of the object and those that are not. Here, we refer to the former as real axes of symmetry and to the latter as virtual axes of symmetry. From the schematic insets above Figure 1, it can be seen that indirect effects can be conceptualized not only as attraction effects toward an almost orthogonal real-line axis of symmetry but also as repulsion effects from a nearby virtual axis of symmetry of that inducing stimulus (dashed lines in insets). Since both of these descriptions of indirect effects are nothing but that (i.e., descriptions), either one is acceptable, although reasons for preferring the latter as a step toward a possible explanation are as follows. First, this way of conceptualizing the indirect effect attributes to virtual axes the property of acting as real, but weak, lines and simultaneously allows both direct and indirect effects to be regarded as repulsion effects, which adds an element of parsimony. Second, the idea that virtual-axis effects are weaker than real-axis effects easily accounts for the broader tuning width of the direct effect, relative to that of the indirect effect. Typically, the angu- 


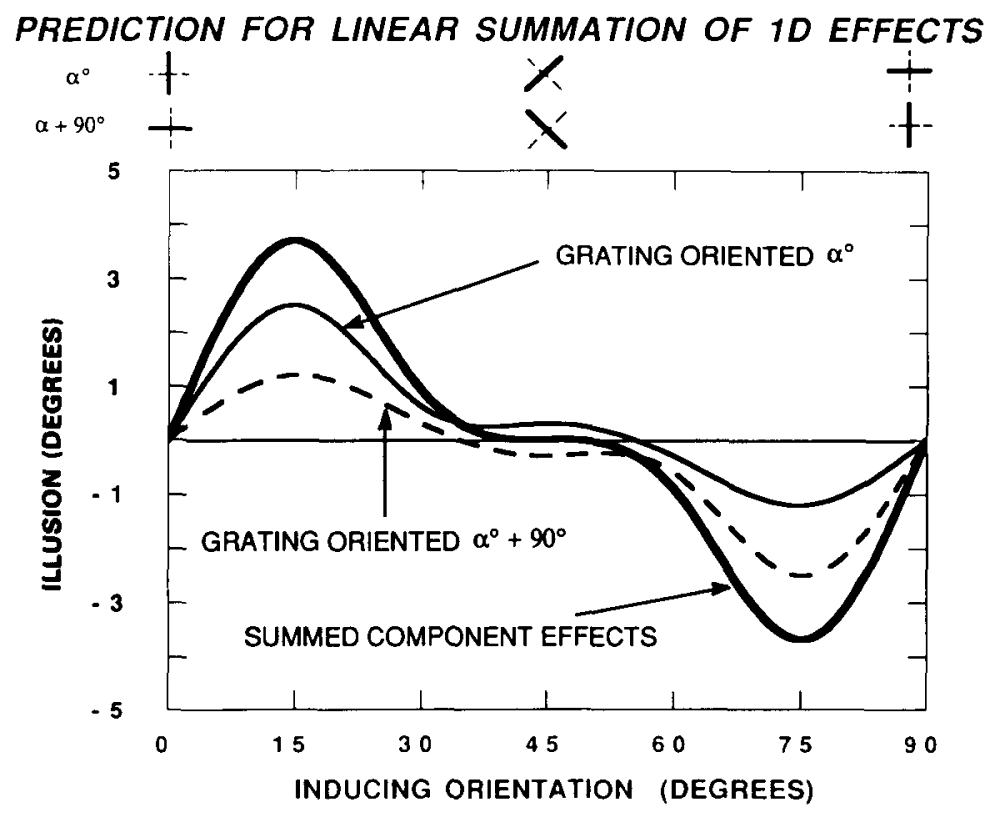

Figure 2. Simple linear sum (heavy line function) of the two 1-D functions in Figure 1.

lar function is found to have its zero crossing at an orientation in excess of $45^{\circ}$, as in Figure 1, and one of the problems with Gibson's "normalization" theory of the tilt aftereffect was that it predicted equal-magnitude direct and indirect effects with the zero crossing at precisely $45^{\circ}$. Third, Wenderoth and Johnstone (1987, 1988a, 1988b) proposed that direct effects arise in V1, whereas indirect effects arise in extrastriate cortex where receptive fields are larger, where cells' responses often are relatively insensitive to spatial position or spatial frequency, and where it has been proposed that mechanisms for the achievement of global orientation constancy reside (see General Discussion). It is not implausible that virtual axes of symmetry are involved in signaling the orientation of complex patterns and that a square frame surrounding an indirect tilt illusion display provides additional information to global orientation mechanisms, overriding the illusory effect of the virtual axis of symmetry by eliminating its influence.

With this conception of an indirect effect as a repulsion from a virtual axis in mind, we shall refer to direct effects as real-axis effects and to indirect effects as virtualaxis effects in what follows. We now ask the question: Given the angular functions of tilt illusions (and aftereffects) schematically represented in Figure 1 , what might be the predicted angular function if both the gratings, that at $\alpha^{\circ}$ and that at $\left(\alpha^{\circ}+90^{\circ}\right)$, were simultaneously presented as inducing stimuli? That is, what if the test stimulus remained a vertical grating but the surrounding inducing stimulus was a plaid made up of superimposed, orthogonal gratings?

The simplest assumption is that the effects of the two orthogonal inducing gratings would show superposition: they would sum in linear fashion. If that were the case, the heavy line in Figure 2 shows the predicted angular function of the plaid-induced illusion. What might be the further predictions if the inducing plaid was surrounded by a square frame, as in Figure $3 \mathrm{~A}$, or separated from the test grating by an annulus gap, or both, as in Figure 3B?

If, as Wenderoth and Johnstone (1987, 1988a, 1988b) claim to have shown, a square frame surrounding a tilt illusion display selectively eradicates virtual-axis effects

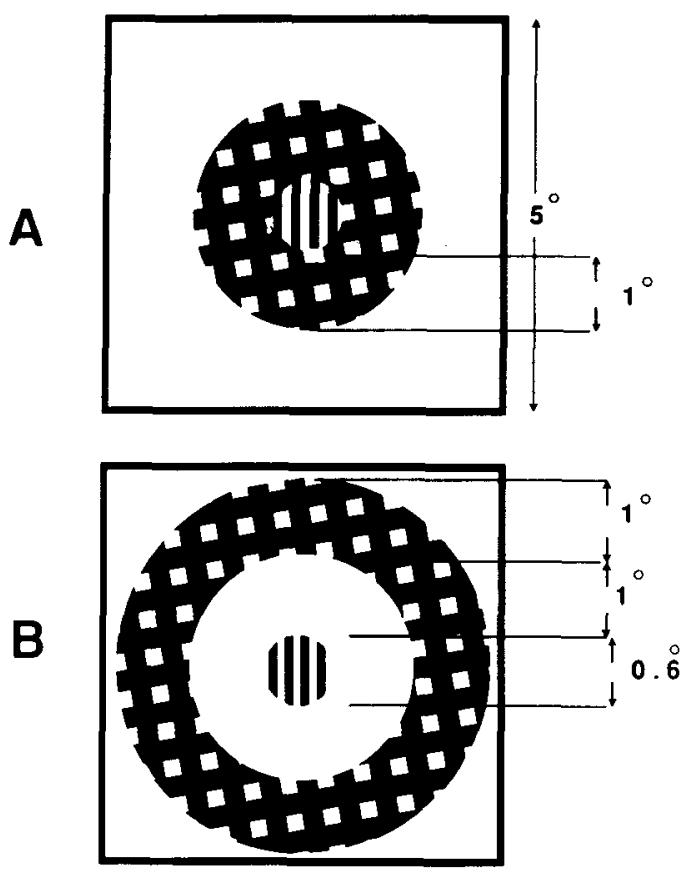

Figure 3. Schematic representation of test grating, inducing plaid, and surrounding square frame when inducing and test stimuli abutted (A) or when there was a $1^{\circ}$ annulus gap (B). 


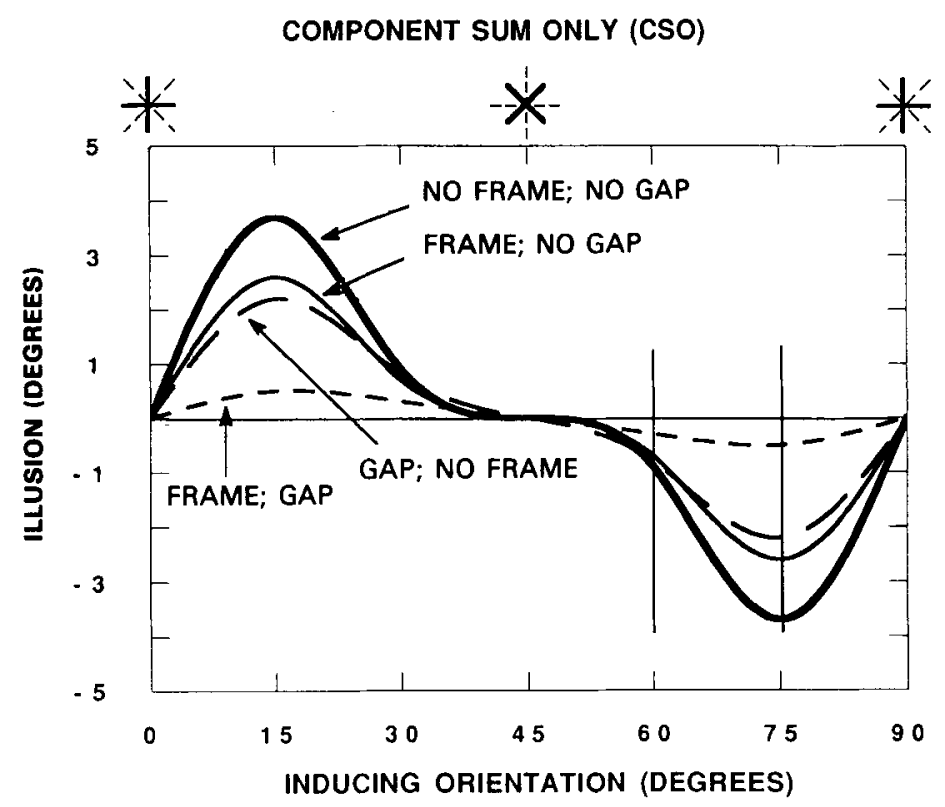

Figure 4. Summed 1-D component illusions for no-frame/no-gap, frame/nogap, no-frame/gap, and frame/gap conditions.

and if a gap between test and inducing stimuli markedly reduces direct effects, then Figure 4 shows the predicted relative effects of these manipulanda on the summed component angular function of Figure 2. The square frame removes the virtual-axis effect, the gap reduces the realaxis effect by an amount slightly larger than the virtualaxis effect (Wenderoth \& Johnstone, 1988a), and the joint presence of both gap and square frame reduces all effects almost to zero.

Let us now consider predictions other than the simple linear summation predictions outlined above and summarized in Figure 4. Consider Figure 5. It presents, schematically, a vertical test grating surrounded by a tilted inducing plaid. If the plaid arbitrarily is considered to be vertical when one of its component gratings is vertical, and if counterclockwise (CCW) tilts are called negative and clockwise (CW) tilts are called positive, then the plaid in Figure 5A is oriented $+75^{\circ}$ (or $-15^{\circ}$ ) and the plaid in Figure $5 \mathrm{~B}$ is oriented $+60^{\circ}$ (or $-30^{\circ}$ ), as indicated in the figures. The point to note in these figures is that once the plaid has been formed by superimposing the two component gratings, it is no longer described adequately as two orthogonal real-line gratings, each with a virtual axis of symmetry orthogonal to its real contours (i.e., as in the insets of Figures 1-5). Rather, the two axes of symmetry of the gratings that were previously virtual are now aligned with the orthogonal real contours and thus are no longer virtual; however, two new virtual axes of symmetry now lie along the bisectors of the component gratings. In Figure 5A, the real, no-longer virtual, axes are oriented $-15^{\circ}$ and $+75^{\circ}$ (solid spokes), and the virtual axes are oriented $-60^{\circ}$ and $+30^{\circ}$ (dashed spokes); in Figure 5B, the respective orientation pairs are $\left(-30^{\circ},+60^{\circ}\right)$ and $\left(-75^{\circ},+15^{\circ}\right)$
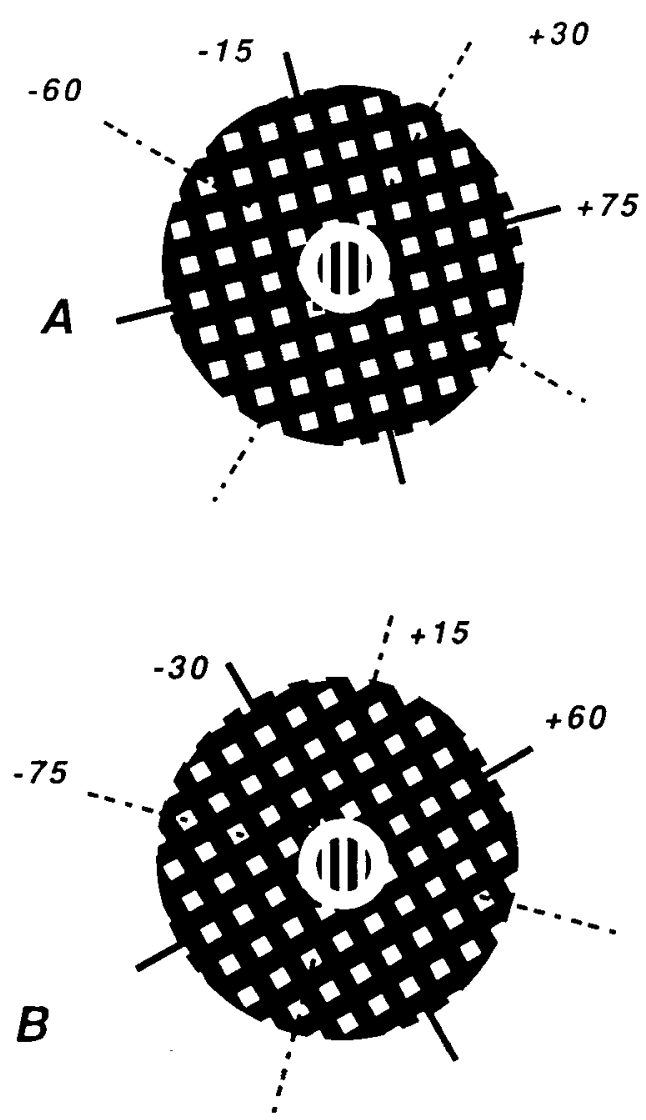

Figure 5. Single, central, and vertical test gratings surrounded by inducing 2-D plaid stimuli. Real plaid components oriented $(A)+75^{\circ}$ and $-15^{\circ}$ with virtual angle bisectors and axes of symmetry at $+30^{\circ}$ and $-60^{\circ}$, and $(B)+60^{\circ}$ and $-30^{\circ}$ with virtual axes of symmetry at $+15^{\circ}$ and $-75^{\circ}$. 
In the light of earlier analysis of 1-D tilt illusions induced by real and virtual axes, it seems logical to propose that, as an inducing plaid tilts from, say, $0^{\circ}$ to $+90^{\circ}$, illusory repulsions of the test grating will be induced not only by the real component gratings but also by one of the plaid's virtual axis of symmetry as it passes through vertical.

The implications of this argument are shown in Figure 6 , in which the inset above the figure schematically represents the real lines of the $2-\mathrm{D}$ inducing stimulus and their virtual bisectors. The dashed function is the same as the heavy function in Figure 4, that is, the linear sum of the component-grating 1-D real- and virtual-axis effects. The dotted function with zero crossing at $45^{\circ}$ is the proposed additional virtual-axis effect. As the positive illusion induced by the real grating tilted $\mathrm{CW}$ of vertical begins to decline around $20^{\circ}-25^{\circ}$ from vertical, the $\mathrm{CCW}$ virtual axis begins to induce a dominant negative illusion. Both functions pass through zero illusion at $45^{\circ}$ because the real grating contours are symmetrical about vertical and the virtual axis is at vertical. After that, the virtual axis is $\mathrm{CW}$ of vertical and induces a positive illusion that dominates until, around $65^{\circ}-70^{\circ}$ tilt, the real $\mathrm{CCW}$ grating begins to induce a dominant negative effect as it now becomes the closer axis to vertical.

Assuming linear summation of the component-grating effects and the virtual-axis effects, the net result is shown by the solid, heavy function in Figure 6. This analysis, then, predicts that the 2-D (plaid-induced) orientation illusion will exhibit an S-shaped angular function similar to the 1-D effect-however, the latter occurs over a $90^{\circ}$ range, whereas the similarly shaped 2-D illusion function will occur over a $45^{\circ}$ range (or twice over a $90^{\circ}$ range).
Given these two models of plaid-induced orientation illusions, one assuming the simple linear sum of component 1-D effects (the component-sum-only, or CSO, hypothesis) and the other assuming component effect summation plus a virtual-axis effect (the component-plusvirtual, or CPV, hypothesis), what does each predict about the effects on 2-D illusions of spatial differences between test and inducing stimuli and a square frame surrounding the entire display?

From Figures 4 and 7 , it can be seen that both models predict the largest effects of spatial manipulations at plaid orientations of $75^{\circ}$ (or $15^{\circ}$ ) but that the effects predicted by the two models are similar. On the other hand, the predictions differ most at $60^{\circ}$ (or $\left.30^{\circ}\right)$. The vertical lines in Figures 4 and 7 , which are drawn at $60^{\circ}$ and $75^{\circ}$ on the abscissa, are at the two orientations of the plaids shown in Figure 5. For each of the CSO and CPV hypotheses, then, the predicted effect of a square frame surrounding each of the entire displays and/or the effect of a gap between the test and inducing stimuli can be read off Figures 4 and 7 at the $+75^{\circ}$ and $+60^{\circ}$ points along the $a b$ scissa. Although the predictions are almost identical in arbitrary magnitude units for the two hypotheses in the $75^{\circ}$ frame, gap and frame-plus-gap conditions, the directional difference in the $+60^{\circ}$ effects makes the predictions for the two hypotheses so different.

The CSO hypothesis predicts that at both the $+75^{\circ}$ and the $+60^{\circ}$ plaid orientations, the initial illusion will be negative and will be reduced by the frame, more so in the gap condition than in the abutting condition. The CPV hypothesis also predicts that at the $+75^{\circ}$ orientation, the initial effect will be negative and will be reduced by the

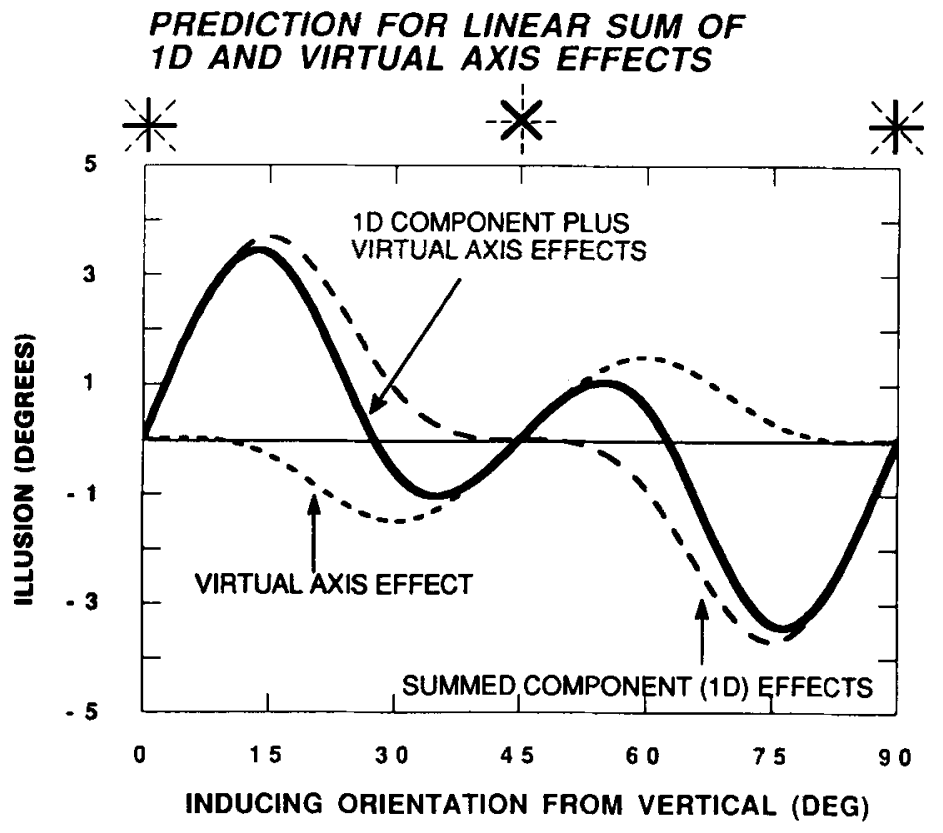

Figure 6. Linear sum (solid function) of summed component 1-D effects (dashed line) and hypothesized virtual-axis effect (dotted line). 
COMPONENT PLUS PURELY VIRTUAL AXIS SUM (CVS)

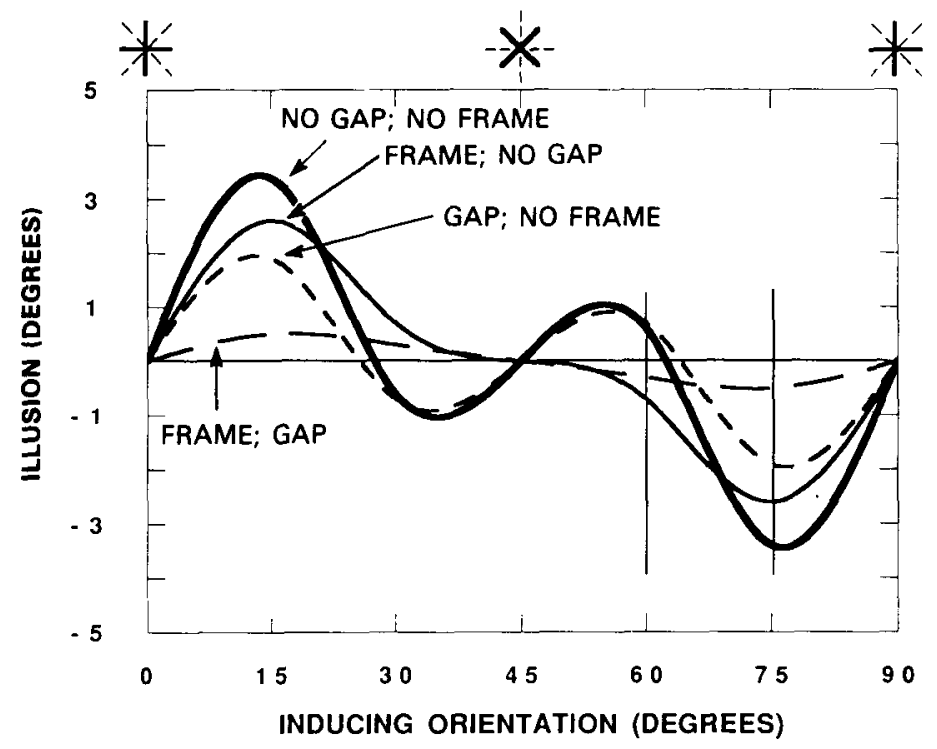

Figure 7. Predicted 2-D plaid orientation illusion angular functions based upon linear sum of component 1-D effects and the linear sum of the virtualaxis effect.

frame, more so in the gap condition than in the abutting condition. However, it predicts that, at $+60^{\circ}$, an initially positive effect will have its direction reversed by the frame, more so in the no-gap condition than in the gap condition.

Experiments 1 and 2 were designed to test these predictions. Experiment 1 tested the effect of a surrounding square frame on 2-D effects induced by the $+75^{\circ}$ plaid in Figure 5A. In this experiment, too, either the annulus plaid abutted the test grating or there was a $1^{\circ}$ annulus gap between them. Also, the inducing plaid components had either the same spatial frequency as the test 5 cycles per degree [cpd]) or half that frequency. It should be stressed that the illusion in Figure $5 \mathrm{~A}$ is such that the vertical test grating appears tilted $\mathrm{CW}$ and is therefore set $\mathrm{CCW}$ to appear vertical. Thus, the convention adopted here-that $\mathrm{CCW}$ errors are negative and $\mathrm{CW}$ errors are positive-means that illusions in the expected direction will be negative.

\section{EXPERIMENT 1}

\section{Method}

Apparatus and Stimuli. Stimulus displays were presented on the flat screen of a Tektronix 608 display monitor (P31 phosphor), interfaced with an Innisfree ("Picasso") Image Generator and a PDP-11/73 minicomputer. The subjects used the outer pair of three microswitches to indicate whether a circular $0.6^{\circ}$ visual angle sinewave test grating appeared tilted left or right of perceived vertical. The experimenter and the subject were located in adjacent laboratories. Intrasession communication was possible via an intercom system; a slave monitor in the experimenter's cubicle enabled monitoring of the subject's judgments. The subjects viewed the display in a dark, windowless cubicle. All external cues to vertical were removed by attaching to the screen a flat black aluminum mask in which a $6.75^{\circ}$ diameter hole had been cut and by draping black cloth over the area between the screen and the subject's headholder. The subject's head rested in a padded forehead-, temple-, and chinrest such that $1 \mathrm{~cm}$ on the screen, $57 \mathrm{~cm}$ from the subject, subtended $1^{\circ}$ of visual angle.

The Image Generator was modified to run automatically via the minicomputer and a custom designed D/A interface. This allowed up to four different screens to be interleaved at a rate of $188 \mathrm{~Hz}$, and software enabled these screens to be constructed using an onscreen menu. So, for example, the inducing annulus containing crossed gratings was constructed by interleaving two of the four screens-one containing one grating within an annulus, the other containing the orthogonal grating within an identically positioned annulus. The rate of interleaving ensured that the display was not seen to flicker. The central test grating always had a spatial frequency of $5 \mathrm{cpd}$. The maximum luminance of the light bars was $13.2 \mathrm{~cd} / \mathrm{m}^{2}$ and the minimum luminance of the dark bars was $2.5 \mathrm{~cd} / \mathrm{m}^{2}$, measured on a low-frequency grating with a Tektronix $\mathrm{J} 161^{\circ}$ digital luminance probe. Thus, test grating Michelson contrast, defined as $\left(\left[L_{\max }-L_{\min }\right] /\left[L_{\max }+L_{\min }\right]\right)$, was 0.68 . Each crossed inducing grating had the same luminance value as the test, but because of luminance summation at their intersects, plaid contrast was higher-namely, 0.81 . When present, the surrounding square frame, $5^{\circ}$ on a side, was defined by luminance edges and was displayed on the fourth screen. Internally, it had a uniform luminance of $2.5 \mathrm{~cd} / \mathrm{m}^{2}$, whereas its surrounds had a luminance of $13.2 \mathrm{~cd} / \mathrm{m}^{2}$. The edges of the square were thus defined by a single increment in luminance at and outside its edges. When the square was absent, the entire frame was blanked at $2.5 \mathrm{~cd} / \mathrm{m}^{2}$. Hence, the contrasts of all test and inducing components did not vary with the presence or absence of the frame. During intertrial intervals (2 sec), the screen was blanked at $6.8 \mathrm{~cd} / \mathrm{m}^{2}$, sufficient to eradicate afterimages between conditions. However, to ensure that the subjects fixated in the center of the test grating, a small dark spot was centered in the blank screen between trials. The inducing annulus containing the plaid was always $1^{\circ}$ thick. Consequently, when it abutted the $0.6^{\circ}$ test grating, its inside diameter was $0.6^{\circ}$ and its outside diameter was $2.6^{\circ}$. In the nonabutting conditions, the annulus gap was $1^{\circ}$ thick: the inducing annulus then had an inside diameter of 
$2.6^{\circ}$ and an outside diameter of $4.6^{\circ}$. Stimulus flash duration was $405 \mathrm{msec}$. The four consecutive screens were presented in the order: test, plaid component 1 , plaid component 2 , frame. Since each screen had a duration of $5.33 \mathrm{msec}$, this sequence was repeated 19 times. We have demonstrated previously, in control experiments, that the ordering of the screens within an interleaving segment makes no significant difference to the illusions obtained (Wenderoth, van der Zwan, \& Johnstone, 1989b).

Procedure. Each subject was tested under 8 experimental conditions: same/different frequency $\times$ square/no-square $\times$ gap/nogap. However, for each of these conditions, there was a matched pretest control in which the test flash occurred for the appropriate duration but no plaid was presented. Whenever a square frame $\alpha c-$ curred in the test condition, it was also present in the matched pretest condition. These 16 conditions were presented in quasirandom order: the abut/gap conditions were sequential in the order abut/gap or gap/abut, with this ordering random. The pretest for each of the eight test conditions always preceded it immediately. Each of the 16 measures of the point of subjective vertical was obtained from a single staircase, which commenced with the test grating oriented randomly within $10^{\circ}$ of vertical. Step size was $2.12^{\circ}$ initially but was reduced to $1.06^{\circ}$ after the first two reversals. In all, 10 reversals were run, and the PSV estimate was based upon the last 6 . Between staircases, the subjects rested for $2 \mathrm{~min}$; the entire experiment lasted about 50-60 min. The instruction to the subjects was simply to judge the direction of deviation of the test grating from vertical. No mention was made of the presence or absence of a surrounding square frame.

Subjects. There were 17 subjects, volunteers from an introductory course who were given nominal course credit in return. All were naive about the experiment, and all had emmetropic or suitably corrected vision.

\section{Results}

Figure 8 shows the results of the pretest control conditions. Although it has panels for same-frequency (left panel) and different-frequency (right panel) conditions as

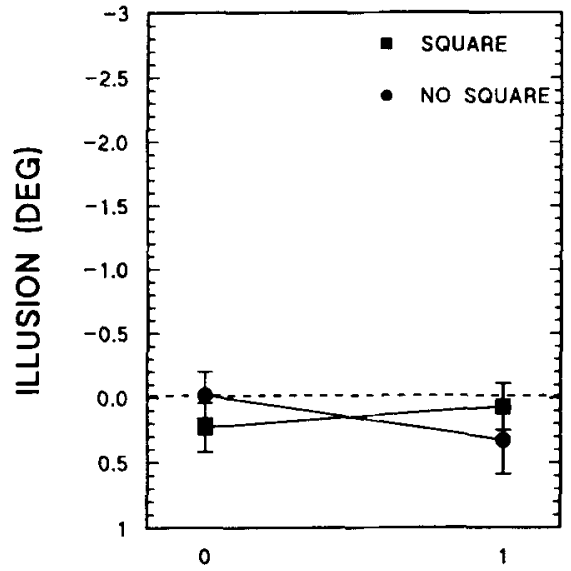

GAP SIZE (DEG)

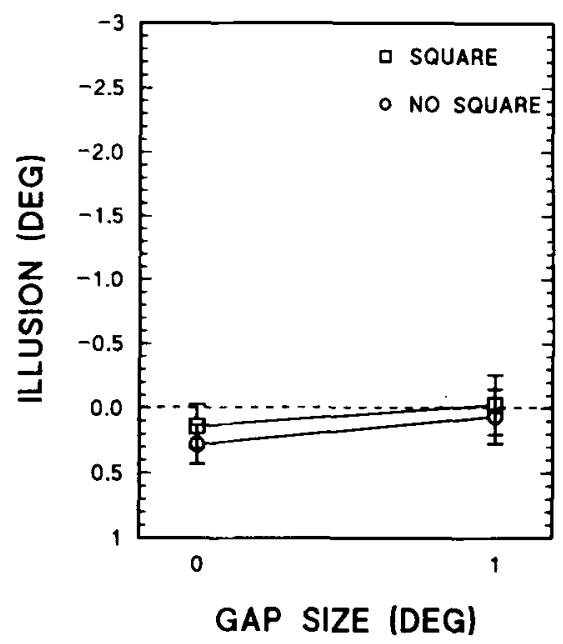

Figure 8. Pretest mean points of subjective vertical under same (left panel, flled symbols) and different (right panel, open symbols) test-inducing spatial-frequency conditions in Experiment 1.
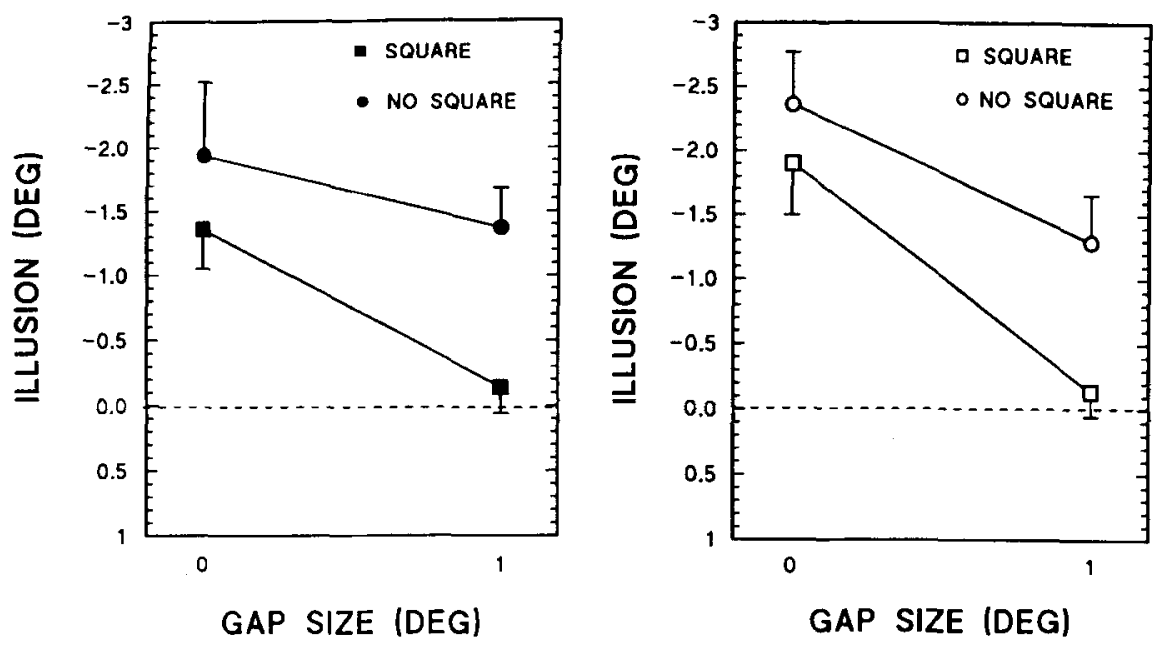

Figure 9. Test-minus-pretest $+75^{\circ}$ plaid illusion measures in Experiment 1 . Conventions as in Figure 8. 
well as gap and no-gap conditions, these are dummy variables for the pretests, because no inducing plaid was present. As is made clear by the figure, there was no systematic bias introduced into pretest settings by the presence of the square frame.

The main results of Experiment 1 are shown in Figure 9 , in which the left panel shows the data from the same-frequency conditions and the right panel gives the different-frequency data. Circles represent no-square conditions, and square symbols indicate the presence of the surrounding frame. All means shown are test-minuspretest illusion measures.

The total treatments variance was broken down into seven comparisons of interest, chosen beforehand, uncorrelated and each with one degree of freedom-that is, the data were analyzed as a simple subject $\times$ treatments design with seven planned orthogonal contrasts. The Type I error rate was set at $\alpha=.05$. Four contrasts tested the differences between pairs of means vertically aligned in Figure 9 (i.e., between the mean pairs that differed only on the square/no-square treatment). In the $1^{\circ}$ gap conditions, both the same-frequency and the different-frequency pairs were significantly different $[F(1,112)=7.78$ and 6.88 , respectively, $p<.01]$. In the abutting conditions, neither pair was different $[F(1,112)=1.76$ and 1.11 , $p>.05]$. Two additional contrasts showed that the effect of gap size was significant, for both the same-frequency condition $[F(1,112)=8.26, p<.005]$ and the differentfrequency condition $[F(1,112)=20.77, p<.0005]$. Finally, there was no overall effect of spatial frequency $[F(1,112)=1.04, p>.05] .{ }^{1}$ The two means in gap/square conditions were not different from zero $[t(16)$ $=0.47$ and $0.46, p>.05]$. All other means were different from zero (all $p s<.001$ ].

These obtained data, then, match the predictions of both the CSO and the CPV hypotheses: the square frame reduced effects at $+75^{\circ}$ plaid tilt, more so (i.e., significantly only) in the gap condition.

\section{EXPERIMENT 2}

There were two differences between Experiments 1 and 2. First, the inducing plaids were oriented $30^{\circ} \mathrm{CCW}$ and $60^{\circ} \mathrm{CW}$, as in Figure 5B. Second, we increased exposure duration in Experiment 2 to $1,620 \mathrm{msec}$ in order to ensure that we avoided confounding effects of duration with the effect, if any, of the frame. We have found (Wenderoth, van der Zwan, \& Johnstone, 1989b) that as exposure duration is decreased from 405 to $15 \mathrm{msec}, 2-\mathrm{D}$ effects at the $+75^{\circ}$ orientation increase markedly in magnitude; however, at the $+60^{\circ}$ orientation, the effects reverse direction, as if virtual axes are not extracted at short durations and component real-axis effects simply sum. This crossover was found to occur at an exposure duration less than $405 \mathrm{msec}$, the duration used in Experiment 1. However, in the present experiment, the illusion stimuli would occupy only $304 \mathrm{msec}$ of a 405 -msec flash, because we introduced a fourth field containing the square frame. For this reason, we increased the duration until we obtained a reliable positive illusion.

\section{Method}

Procedure. All aspects of method and procedure were the same as in Experiment 1, except for the orientations of the plaid components and stimulus duration. The $1,620-\mathrm{msec}$ flash consisted of the four interleaved screens (test, plaid component 1, plaid component 2 , and frame) repeated 76 times.

Subjects. There were 19 subjects, drawn from the same population as those in Experiment 1.

\section{Results}

The pretest control means are shown in Figure 10, in similar fashion to those in Figure 8 for Experiment 1 . Although they were slightly more positive in this case, there is again no systematic effect of the presence of the square frame.

The test-minus-pretest means are shown in Figure 11 on an expanded $y$-axis scale, because effects induced at
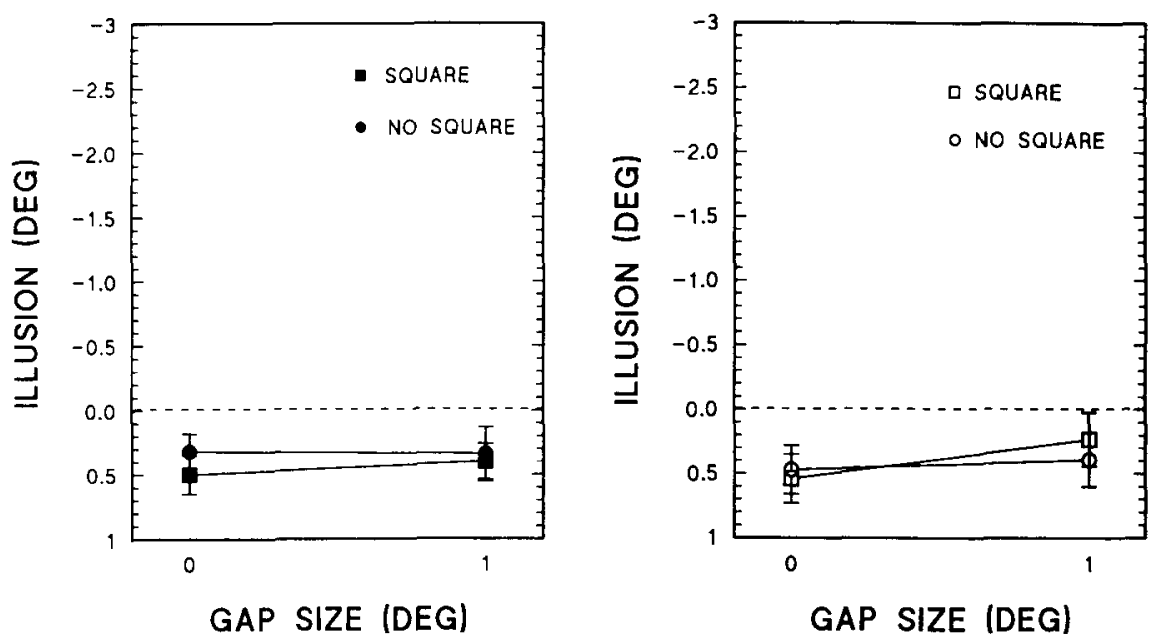

Figure 10. Pretest means in Experiment 2. Conventions as in Figure 8. 


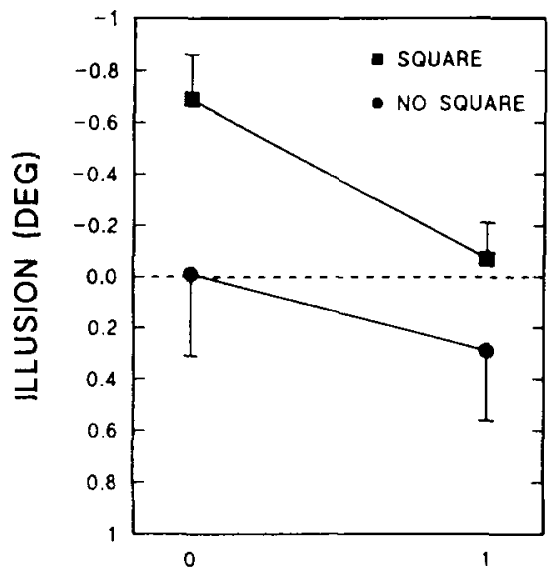

GAP SIZE (DEG)

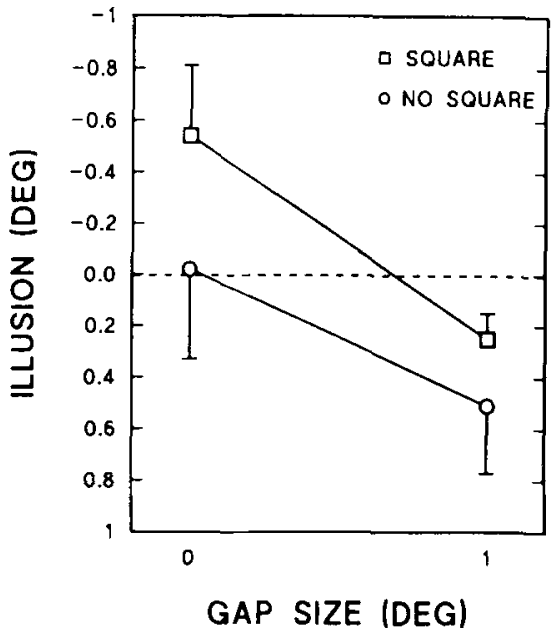

Figure 11. Test-minus-pretest $+60^{\circ}$ plaid illusion measures in Experiment 2. Conventions as in Figure 8.

this plaid orientation were small. Analysis was again by seven orthogonal contrasts, the same set used in Experiment 1 . For the same-frequency data (left panel), the zerogap means for the square present and absent were different $[F(1,126)=5.60, p<.025]$. These means were not different in the $1^{\circ}$ gap conditions $[F(1,126)=1.59$, $p>.05]$. For the different-frequency data (right panel), the $0^{\circ}$ gap means were close to differing significantly, but did not $[F(1,126)=3.27, .1>p>.05]$. The $1^{\circ}$ gap means were not different $[F(1,126)=0.80$, $p>.05]$. The effect of the gap was significant in both the same-frequency conditions $[F(1,126)=5.03, p<.025]$ and the different-frequency conditions $[F(1,126)=10.45$, $p<.005]$. The overall effect of spatial frequency was not significant $[F(1,126)=1.41, p>.05]$. Three of the means in Figure 11 were significantly different from zero: the two square-present, zero-gap means (for the left- and right-panel means, respectively, $t \mathrm{~s}=3.39$ and 2.65 , $p s<.01$ and .02$)$. The other significant mean was that for the square-absent effect in the different-frequency/gap condition $(t=2.50, p<.05)$. In the absence of the frame, then, the sole significant plaid-induced effect was positive, as predicted by the CPV hypothesis but not by the CSO hypothesis. Also as predicted by the former but not the latter hypothesis, and as is clear from Figure 11, the square frame reduced effects (i.e., made them more negative) more in the zero-gap condition than in the $1^{\circ}$ gap condition: Despite the fact that only one of the two comparisons of the zero-gap means reached significance, the fact that the frame produced significant negative illusions in both of the abutting conditions but not in the gap conditions is consistent with the CPV predictions.

\section{GENERAL DISCUSSION}

The experiments reported here add to the considerable body of evidence, discussed below, that the visual sys- tem extracts virtual axes of symmetry from patterns and that these axes act as weak, but real, lines. Using stimulus displays that induce what we have described as 2-D real-axis effects (Figure 5A) and 2-D virtual-axis effects (Figure 5B), Experiments 1 and 2 have demonstrated that a square surrounding frame significantly reduces the 2-D real-axis effect, but only when there is a gap between test and inducing stimuli, and that the frame significantly reverses the direction of the 2-D virtual axis effects, but only when there is no gap between test and inducing stimuli. These outcomes were not predicted by the hypothesis that 1-D (or single-grating) tilt illusions simply sum when the inducing gratings are added to form an inducing plaid (the CSO hypothesis). They were predicted by the hypothesis that the plaid induces linearly summed 1-D illusions plus the effects of a virtual axis of symmetry of the plaid, located along the bisector of the plaid's component gratings (the CPV hypothesis).

When first we reported that spatial differences between test and inducing components of 1-D orientation illusions reduced real-axis effects but not virtual-axis effects and that a surrounding remote square frame reduced virtualaxis effects but not real-axis effects, we concluded that the two effects had different mechanisms (Wenderoth \& Johnstone, 1987, 1988a). We speculated that real-axis effects might reflect the operation of lower level mechanisms in V1, where neurons generally are tightly tuned to spatial position and frequency, but that virtual-axis effects might arise at a higher level in extrastriate cortex, where receptive fields are larger and often not so specifically tuned (e.g., Allman, Miezin, \& McGuinness, 1985; Desimone, Schein, Moran, \& Ungerleider, 1985).

Remote interactions between a surrounding square frame and the virtual-axis illusion could have their basis in long-range connections between isooriented cells in V1 (e.g., T'so, Gilbert, \& Wiesel, 1986). Such cells have total receptive fields (TRFs) that are larger than classical 
receptive fields (CRFs) and whose CRF properties are modulated by remote stimuli, which, when presented alone, have no effect on the CRF (Allman et al., 1985). Thus, such cells may be involved in the remote interactions we describe and may be located either in extrastriate cortex or in V1 with feedback from extrastriate areas (Allman et al., 1985; Maunsell \& Newsome, 1987; Nelson \& Frost, 1978). However, the fact that large-scale interactions occur in extrastriate visual areas, with receptive fields often extending across the midline (unlike V1), and the fact that cells in these areas often respond to complex patterns suggest an extrastriate neural basis for global perceptual mechanisms mediating not only pattern perception but also orientation constancy, subjective contours, figure-ground segregation, and depth perception through motion (Allman et al., 1985; Heydt \& Peterhans, 1989; Maunsell \& Newsome, 1987; Movshon, Adelson, Gizzi, \& Newsome, 1987; Peterhans \& Heydt, 1989). We therefore offered as plausible the hypothesis that the 1-D virtual-axis effect could be seen as arising from global extrastriate mechanisms involved in orientation constancy. Nevertheless, given that TRFs have been reported in V1, and bearing in mind that previously reported V1 CRFs may reflect more about the biased and selective use of certain probe stimuli by the investigators than about underlying structure and function, it may be prudent to refer to local (real-axis) and global (virtual-axis) effects as arising from CRF and TRF interactions, respectively, rather than attempting to localize these respective effects in striate and extrastriate cortex. This view is strengthened by the fact that similar global interactions to those found in extrastriate areas-that is, large TRFs-have now been found in V1 and V2 (Allman, personal communication, September 1990).

Wenderoth and Johnstone $(1987,1988 \mathrm{a})$ proposed that the rich array of orientation cues normally available in the environment minimizes orientation illusions under normal circumstances. In the laboratory, under impoverished conditions, illusions do occur, except when the remote surrounding frame provides the global orientation mechanism with additional orientation data (Wenderoth \& Johnstone, 1988a). The experiments reported here provide a much stronger test of the above ideas. We have noted elsewhere that the putative local and global contributions to orientation illusions " may be evidenced only under conditions where the two mechanisms compete and the magnitude of the response of one is selectively manipulated" (Johnstone \& Wenderoth, 1989). By using 2-D plaidinduced illusions, we have deliberately chosen stimulus displays in which both real and virtual axes of symmetry closely flank vertical (i.e., are not orthogonal). We have used gaps between test and inducing stimuli to manipulate (reduce) the hypothesized V1 contribution to the illusions induced by real axes, and we have used a surrounding vertical frame to manipulate (reduce) the hypothesized extrastriate contribution to the illusions induced by solely virtual axes. By analyzing the expected effects of the joint manipulation of gap and frame presence or absence in a single experiment (i.e., Experiment 1), we were able to predict, from both the CSO and the CPV hypotheses, that the surrounding frame would reduce 2-D orientation illusions at $+75^{\circ}$ plaid tilt, but to a greater extent with a gap present. However, only the CPV hypothesis predicted that the initial effect at $+60^{\circ}$ plaid tilt would be positive and that the frame would tend to reverse the direction of 2-D effects at $+60^{\circ}$ plaid tilt, more so with no gap present.

In fact, we have previously reported that illusions induced by orthogonal 1-D inducing stimuli are not simply additive (Wenderoth, 1977; Wenderoth \& Beh, 1977; Wenderoth \& Curthoys, 1974). We have also reported on plaidinduced illusions with and without test-inducing gaps and spatial-frequency differences. We have measured the complete angular function of the 2-D orientation illusion and obtained functions similar to that in Figure 7 (Wenderoth, Johnstone, \& van der Zwan, 1989). What is new in the present paper is the quantitative predictions in Figures 1-7, rather than the more qualitative analysis adopted previously, and we have never before used a surrounding frame with a 2-D illusion. For example, we previously reported that gaps between the test grating and an inducing plaid reduced illusions at $+75^{\circ}$ plaid tilt but increased the effects at $+60^{\circ}$ plaid tilt (Johnstone \& Wenderoth, 1989; Wenderoth, Johnstone, \& van der Zwan, 1989; Wenderoth, van der Zwan, \& Johnstone, 1989a, 1989b). We proposed that, whether there is a gap or not, the effect obtained at $+60^{\circ}$ is due largely to the dominant repulsion from the virtual axis at $+15^{\circ}$ (Figure 5B). However, we argued, this effect is somewhat offset by the directionally opposite effect of the real axis at $-30^{\circ}$. Introducing a gap between the inducing and test stimuli reduces the effect of the real 1-D axis (Wenderoth \& Johnstone, 1988a) and so appears to increase the virtual-axis effect by subtracting less from it. The basis of this explanation can be seen graphically here in Figure 7: At $+60^{\circ}$, the magnitude of the summed illusion components does increase slightly with the introduction of a gap.

Interestingly, in earlier reports, we have pointed to the similarity, in terms of inducing axes, of the 2-D orientation illusion and the rod-and-frame effect (Wenderoth, Johnstone, \& van der Zwan, 1989). DiLorenzo and Rock (1982) studied the rod-and-frame effect when a vertical rod and tilted frame were embedded in a larger vertical square frame and when a vertical rod and vertical frame were surrounded by a large tilted frame. They found that the illusion of rod tilt was eradicated in the first case but not the second and attributed the results to perceptual problem solving. We would argue that the first result mirrors our finding of a large 2-D direct effect reduction in the $1^{\circ}$ gap condition with the surrounding frame, and that the second result indicates that the large peripheral tilted frame induced a global tilt in both the truly vertical, central rod and the frame.

The data reported here can therefore be taken as strong evidence for the role of virtual axes of symmetry in orientation processing. One possible explanation of virtual-axis effects is that lateral inhibition in the orientation or position 
domain, which is propagated by each component grating, summates along the bisector between the gratings, a possibility considered by Wenderoth (1977) and Wenderoth and Beh (1977). However, the lack of spatial position tuning of virtual-axis effects renders such an explanation unlikely. Apart from our own evidence to that effect, Hartley (1982) demonstrated a series of orientation illusions in which test and inducing components were separated widely in space $\left(1^{\circ}-6^{\circ}\right)$, with no diminution in the illusions as separation increased. He concluded:

A plausible explanation is that axes of symmetry, extracted by interactions in the orientation domain that operate over broad areas of the retinal field, have perceptual consequences much like physically present facets of the retinal image.... Symmetry discrimination would be part of a global texture perception system in contrast to form recognition, which is a local scrutiny system relying on all stages of feature extraction.... Replacing a figure by its axes of symmetry could be used to separate figure from ground, to determine orientation, and to classify input loosely. (Hartley, 1982, p. 375)

Movshon et al. (1985) reported that some cells in extrastriate area MT responded to the motion direction of the component gratings of a drifting plaid, whereas other cells responded only to the pattern direction and not to the component directions, consistent with what is generally perceived. They suggested that the latter "second stage analyzers" might act in an AND-gating fashion by requiring both "first stage analyzers" (cells responding to the components) to be simultaneously active before themselves responding. In a similar vein, we have discussed the possibility that real component orientations are extracted by local mechanisms (V1, CRFs?) but that global pattern orientations are coded, or AND-gated, by more global mechanisms (extrastriate, TRFs?), and it is these that account for the apparent psychophysical salience of virtual axes of symmetry. It now remains to be seen whether visual neurophysiologists will discover the extrastriate AND-gating mechanisms, which, we have postulated, underlie the extraction of global and solely virtual axes of symmetry (Wenderoth \& Johnstone, 1987; Wenderoth, van der Zwan, \& Johnstone, 1989a, 1989b).

It is not inconceivable that virtual and global axes of symmetry are extracted by a combined weighting of local real and virtual axes to produce the axis that, in the context of our experiments, partly determines the magnitude and direction of the pattern-induced orientation illusion but that, in the broader context, in effect defines the perceived orientation of a complex multicontoured pattern.

\section{REFERENCES}

Allman, J. M., Miezin, F., \& McGuinness, E. (1985). Stimulus specific responses from beyond the classical receptive field: Neurophysiological mechanisms for local-global comparisons in visual neurons. Annual Review of Neuroscience, 8, 407-430.
Blakemore, C., Carpenter, R. H. S., Georgeson, M. A. (1970). Lateral inhibition between orientation detectors in the human visual system. Nature, 228, 37-39.

Campbell, F. W., Maffei, L. (1971). The tilt aftereffect: A fresh look. Vision Research, 11, 833-844.

Desimone, R., Schein, S. J., Moran, J., \& Ungerleider, L. G. (1985). Contour, colour and shape analysis beyond the striate cortex. Vision Research, 25, 441-452.

DiLorenzo, J. R., Rock, I. (1982). The rod-and-frame effect as a function of the righting of the frame. Journal of Experimental Psychology: Human Perception \& Performance, 8, 536-546.

Georgeson, M. A. (1973). Spatial frequency selectivity of a visual tilt illusion. Narure, 245, 43-45.

Gibson, J. J. (1937). Adaptation, aftereffect and contrast in the perception of tilted lines: II. Simultaneous contrast and the areal restriction of the aftereffect. Joumal of Experimental Psychology, 20, 553-569.

Gibson, J. J., RADNER, M. (1937). Adaptation, aftereffect and contrast in the perception of tilted lines: I. Quantitative studies. Joumal of Experimental Psychology, 20, 453-467.

HARTLEY, A. A. (1982). The roles of axes of symmetry in orientation illusions. Perception \& Psychophysics, 31, 367-375.

Heydt, R. von der, Peterhans, E. (1989). Mechanisms of contour perception in monkey visual cortex. I. Lines of pattern discontinuity. Joumal of Neuroscience, 9, 1731-1748.

Howard, I. P. (1982). Vismal spatial orientation. New York: Wiley. Johnstone, S., Wenderoth, P. (1989). Spatial and orientation specific integration in the tilt illusion. Perception, 18, 5-23.

Maunsell, J. H. R., \& Newsome, W. T. (1987). Visual processing in monkey extrustriate cortex. Annual Review of Neuroscience, 10 , $363-402$.

Mitchell, D. E., Muir, D. (1976). Does the tilt aftereffect occur in the oblique meridian? Vision Research, 16, 609-613.

Morant, R. B., Harris, J. R. (1965). Two different aftereffects of exposure to visual tilts. American Joumal of Psychology, $\pi, 218-226$.

Movshon, J. A., Adelson., E. H., Gzzz, M. S., Newsome, W. T. (1985). The analysis of moving visual patterns. In C. Chagas, R. Gattass, \& C. Gross, (Eds.), Partem recognition mechanisms (pp. 117-151). Rome: Academis Scientianum.

Nelson, J. I., * Frost, B. J. (1978). Orientation-selective inhibition from beyond the classical visual receptive field. Brain Research, 139. 359-365.

O'ToOle, B., Wenderoth, P. (1977). The tilt illusion: Repulsion and attraction effects in the oblique meridian. Vision Research, 17, 367-374.

Over, R., Broerse, J., \& Crassini, B. (1972). Orientation illusion and masking in central and peripheral vision. Joumal of Experimental Psychology, 96, 25-31

Peterhans, E., * Heydt, R. von der. (1989). Mechanisms of contour perception in monkey visual cortex: II. Contours bridging gaps. Journal of Neuroscience, 9, 1749-1763.

Tolnurst, D. J., * Thompson, P. G. (1975). Orientation illusions and aftereffects: Inhibition between channels. Vision Research, 15, 967-972.

T'so, D. Y., Gilbert, C. D., Wiesel, T. N. (1986). Relationships between horizontal interactions and functional anchitecture in car striate cortex revealed by cross-correlational analysis. Journal of Neuroscience, 6, 1160-1170.

VIRSU, V., TASKINEN, H. (1975). Central inhibitory interactions in human vision. Experimental Brain Research, 23, 65-74.

WALLACE, G. K. (1969). The critical distance of interaction in the Zöllner illusion. Perception \& Psychophysics, 5, 261-264.

WARE, C., MITCHELL, D. E. (1974). The spatial selectivity of the tilt aftereffect. Vision Research, 14, 735-737.

WENDEROTH, P. (1977). An analysis of the rod-and-frame illusion and its variants. In R. H. Day \& G. S. Stanley (Eds.), Studies in perception (pp. 95-141). Perth: University of Western Australia.

Wenderoth, P., BeH, H. (1977). Component analysis of orientation illusions. Perception, 6, 57-75.

WENDEROTH, P., CURTHOYs, I. (1974). On the non-additivity of tilt illusions. Quarierty Joumal of Experimental Psychology, 26, 549-555. 
Wenderoth, P., \& Johnstone, S. (1987). Possible neural substrates for orientation analysis and perception. Perception, 16, 693-709.

WENDEROTH, P., JohNSTONE, S. (1988a). The different mechanisms of the direct and indirect tilt illusions. Vision Research, 28, 301-312.

Wenderoth, P., \& Johnstone, S. (1988b). The differential effects of brief exposures and surrounding contours on direct and indirect tilt illusions. Perception, 17, 165-176.

Wenderoth, P., Johnstone, S., \& VAN DER ZWan, R. (1989). Twodimensional tilt illusions induced by orthogonal plaid patterns: Effects of plaid motion, orientation, spatial separation and spatial frequency. Perception, 18, 471-482.

WENDEROTh, P., VAN der ZwAN, R., a JohnSTONE, S. (1989a). Mechanisms of orientation illusions. In D. Vickers \& P. L. Smith (Eds.), Human information processing: Measures, mechanisms, and models (pp. 83-106). Amsterdam: Elsevier/North-Holland.

Wenderoth, P., Van der Zwan, R., a Johnstone, S. (1989b). Otientation illusions induced by briefly flashed plaids. Perception, 18, 715-728.

\section{NOTE}

1. At first, we were puzzled by the absence of an effect of spatial frequency of the plaid on the illusion. Previously, we have always found that a difference in frequency between test and inducing components reduces the direct effect by about $50 \%$ (Wenderoth, Johnstone, \& van der Zwan, 1989; Wenderoth, van der Zwan, \& Johnstone, 1989b). However, these spatial-frequency effects emerged only at a display duration of $405 \mathrm{msec}$ in an earlier snudy and were not apparent at shorter flash durations (Wenderoth, van der Zwan, \& Johnstone, 1989b). In that study only three, not four, screens were interleaved. In the present case, $25 \%$ of the 405-msec flash contained the square frame/blank screen, so that the test and inducing components were presented for a total of only $304 \mathrm{msec}$. We believe that this accounts for the lack of a spatial-frequency effect.

(Manuscript received November 5, 1990; revision accepted for publication June 3, 1991.)

\title{
Notices and Announcements
}

\author{
Call for Assistance in \\ The Compilation of a History of the Psychonomic Society
}

The Governing Board of the Psychonomic Society is pleased to announce that Robert C. Bolles has agreed to serve as the first Historian of the Society.

All members who might have information relevant to this undertaking are invited to send it directly to Dr. Bolles. Founding members and those who attended the early meetings are especially encouraged to record their reminiscences. While Dr. Bolles hopes to collect as much information as possible relevant to the history of the Society, he will concentrate first on the early history.

Dr. Bolles's address is Department of Psychology, University of Washington, Seattle, Washington 98195 (phone: 206-543-2631). 